\title{
Influence of postoperative adjuvant transarterial chemoembolization on the prognosis of early-stage intrahepatic cholangiocarcinoma: a single center study
}

\author{
Guofang Liu ${ }^{1 \#}$, Wei Guo ${ }^{2 \#}$, Hao Wang ${ }^{1}$, Wendi Liu ${ }^{1}$, Liping Lei ${ }^{1}$, Qiaohua Xie ${ }^{1}$, Xiaoyan Li $^{1}$, \\ Shanshan Zou ${ }^{1}$, Peng Wang ${ }^{1}$, Huabang Zhou ${ }^{1}$, Heping $\mathrm{Hu}^{1}$ \\ ${ }^{1}$ Department of Hepatobiliary Medicine, Shanghai Eastern Hepatobiliary Surgery Hospital, Shanghai, China; ${ }^{2}$ Department of Military Health \\ Statistics, Naval Medical University, Shanghai, China \\ Contributions: (I) Conception and design: G Liu, H Hu; (II) Administrative: W Guo, H Zhou, H Wang; (III) Provision of study material or patients: \\ G Liu, W Guo, H Zhou; (IV) Collection and assembly of data: G Liu, H Wang, W Liu, Q Xie; (V) Data analysis and interpretation: G Liu, W Guo, \\ H Zhou; (VI) Manuscript writing: All authors; (VII) Final approval: All authors. \\ \#These authors contributed equally to this work. \\ Correspondence to: Heping Hu; Huabang Zhou. Department of Hepatobiliary Medicine, Shanghai Eastern Hepatobiliary Surgery Hospital, Shanghai \\ 200438, China. Email: hp-hu@medmail.com.cn; dairydreamer@126.com.
}

Background: The effectiveness of postoperative adjuvant transarterial chemoembolization (TACE) on survival and recurrence in tumor-node-metastasis (TNM) stage I intrahepatic cholangiocarcinoma (ICC) after radical resection remains unclear. This study aimed to compare overall survival (OS) and recurrencefree survival (RFS) in TNM stage I ICC patients with and without postoperative TACE.

Methods: A retrospective cohort study was conducted on TNM stage I ICC patients who had undergone R0 resections with curative intent in Shanghai Eastern Hepatobiliary Surgery Hospital from January 2012 to December 2016. A total of 269 patients were divided into two groups: (I) 35 patients who received postoperative TACE and (II) 234 patients no TACE. Staging was performed according to the 8th edition of American Joint Committee on Cancer (AJCC) TNM staging system. The tumor-related RFS and OS were estimated by the Kaplan-Meier method. Cox proportional regression model was employed to evaluate the prognosis between the two groups.

Results: In all patients, the median OS was 66.8 months. After R0 resection, adjuvant TACE could not improve the survival of TNM stage I patients, and the OS of the TACE group was not better than that of the non-TACE group (P=0.7070). In addition, in the TACE group, the recurrence rate of TNM stage I ICC patients was statistically significantly higher than that of the non-TACE group $(\mathrm{P}=0.0328)$. Multivariable analysis revealed that adjuvant TACE was an independent predictor of worse RFS (HR: 1.88, 95\% CI: 1.212.93).

Conclusions: Adjuvant TACE after radical surgery failed to prolong the OS and potentially delay recurrence for patients with TNM Stage I ICC. Adjuvant TACE might not be suitable for patients with TNM Stage I ICC.

Keywords: Transarterial chemoembolization (TACE); intrahepatic cholangiocarcinoma (ICC); overall survival (OS); recurrence-free survival (RFS)

Submitted Jun 29, 2020. Accepted for publication Dec 28, 2020.

doi: 10.21037/apm-20-1337

View this article at: http://dx.doi.org/10.21037/apm-20-1337 


\section{Introduction}

Intrahepatic cholangiocarcinoma (ICC) is a malignant disease of the biliary tree and the second most frequent primary hepatic malignancy after hepatocellular carcinoma (HCC), accounting for $10 \%$ to $15 \%$ of primary liver cancers (1). Although the frequency of ICC is considerably less than HCC, of note, the incidence and cancer-related mortality of ICC have rapidly increased over the last few decades worldwide (2).

Partial hepatectomy is considered the standard curative treatment option for ICC. However, despite advances in surgical modalities, prognosis after tumor resection remains very poor (5-year survival is only $30 \%$ ), mainly due to the highly malignant biological behavior of ICC, which leads to recurrence early after radical resection (3-6). Previous prospective/retrospective studies have shown that transarterial chemoembolization (TACE) is not only beneficial for patients with intermediate HCC who are not suitable for surgical treatment or local ablation, but also for patients with moderate (tumor size $>5 \mathrm{~cm}$ ) or high risk of HCC after resection Recurrence (single tumor with microvascular invasion; 2 or 3 tumors) (7-9). The basic principle of TACE is that the infusion of cytotoxic agents into the arteries and then embolization of the blood vessels for the tumor will result in strong cytotoxicity and ischemic effects (10). Because HCCs are usually hypervascular, TACE may have anti-tumor effects. However, most ICC lesions are diagnosed with insufficient blood vessels in contrast-enhanced tomography and may exhibit extensive fibrosis, which is often seen at the site of tumor resection. Theoretically, these features may reduce the penetration of the intra-arterial payload into the tumor, thereby reducing the efficacy of TACE on ICC. However, more and more evidences show that TACE can achieve anti-tumor effects in some patients with advanced ICC. A single-center, retrospective cohort study of 155 patients with unresectable ICC reported a significantly longer median overall survival (OS) (12.2 months) in the TACE group than patients in the symptomatic treatment group (11). A recent meta-analysis including a total of 16 articles and 542 patients with unresectable ICC further demonstrated transarterial chemotherapy-based treatments for ICC appeared to confer a survival benefit of 2-7 months compared with systemic therapies (12). Related research also explored the use of TACE as an adjuvant treatment after radical surgery. In a retrospective analysis of 125 ICC patients, it was found that compared with the control group, the survival time of patients receiving TACE treatment was prolonged. The median OS of the adjuvant TACE group was 12 months, while the median OS of surgery was only five months (13). Jeong et al. also found TACE significantly improved the prognosis of patients with HBV-associated ICC (14). However, $\mathrm{Wu}$ et al. found that postoperative adjuvant TACE may only prolong the survival time of ICC patients with tumor size $\geq 5 \mathrm{~cm}$ or advanced TNM stage (15). Another recent retrospective cohort of 553 ICC patients also demonstrated that the TACE and non-TACE groups had similar median OS, 1-, 3-, and 5-year OS rates, and 1-, 3 -, and 5-year tumor recurrence rates using 1:1 propensity score matching method. The study also found that a stratified analysis based on the TNM seventh staging system showed that adjuvant TACE had no significant difference in OS or recurrence-free survival (RFS) in stage I and II patients, but it seemed to improve prognoses in patients at stages III and IV. In terms of OS and disease recurrence rates, there were no significant differences between TACE and non-TACE groups (16). Thus it can be seen that differences in tumor stage may be the primary drivers of disparities in survival and recurrence. And whether any benefit gain could be achieved for earlystage ICC after hepatectomy with adjuvant TACE remains unclear. To address this issue, we conducted a retrospective cohort study to evaluate the prognosis of postoperative TACE in UICC stage I ICC patients.

We present the following article in accordance with the STROBE reporting checklist (available at http://dx.doi. org/10.21037/apm-20-1337).

\section{Methods}

\section{Ethical approval}

The research was conducted in accordance with the Declaration of Helsinki (as revised in 2013). The study was approved by the institutional ethics committee of the Eastern Hepatobiliary Surgery Hospital [K2016001P002(III)]. The committee waived the need for informed consent (both written and oral) from participants because this was a retrospective observational study, involved no more than minimal risk to the subjects and did not include intentional deception; this waiver does not adversely affect the rights and welfare of the patients. 


\section{Patients}

In this retrospective analysis, we collected 269 patients diagnosed with TNM stage I ICC received surgery in Eastern Hepatobiliary Surgery Hospital from January 2012 to December 2016. All patients in this study underwent R0 resection (liver transplantation excluded). The staging of tumors was determined according to the TNM classification system of the 8 th edition. The histological grade of tumor differentiation was assigned by the Edmondson grading system. R0 resection refers to complete resection of all tumors and no tumor cells on the edge of the tumor under microscope. Patients who met the following criteria were enrolled: (I) TNM stage I ICC; (II) liver function of Child-Pugh grade A or B. (III) no evidence of extrahepatic metastasis. (IV) absence of extrahepatic disease. The exclusion criteria are as follows: (I) past or current medical history of other concurrent malignant tumors; (II) recurrent ICC; or (III) radiofrequency ablation (RFA), microwave coagulation therapy (MCT) or cryoablation was performed before surgery. Patients without clinical or imaging followup were also excluded from the analysis.

\section{Clinicopathological factors}

Clinicopathological factors may be related to survival and recurrence were selected in this study, including age, gender, HBsAg (hepatitis B surface antigen) status, cirrhosis, carbohydrate antigen (CA) 19-9, alpha-fetoprotein (AFP), carcinoembryonic antigen (CEA), tumor size (main tumor or the largest one), tumor number (satellite nodules, mutifocal primary cholangiocarcinomas, and intrahepatic metastases are not distinguished and are considered multiple tumors), tumor location, tumor histologic type, tumor capsule formation, tumor differentiation (according to the WHO classification system of tumor: well, moderately or poorly differentiated; when histological diversity was observed in a tumor, the higher grade was taken as the overall grade), vascular invasion (included major portal vein invasion, hepatovein invasion, and microvascular invasion), lymphatic metastasis (included regional lympha node metastasis and distant lympha node metastasis), extrahepatic metastasis (tumor directly metastasized extrahepatic tissues or organs).

\section{Adjuvant TACE after $\mathrm{RO}$ resection}

It is recommended that patients after $\mathrm{R} 0$ resection receive postoperative adjuvant TACE treatment. Patients who decide to receive adjuvant TACE also need to have a WHO performance status of $0-1$, Child-Pugh A or B, white blood cell count $\geq 3.0 \times 10^{9} / \mathrm{L}$, platelet count $\geq 50 \times 10^{9} / \mathrm{L}$, and normal renal function. The adjuvant TACE was performed one time 1-1.5 months after the operation. Adjuvant TACE was performed by injecting $3-5 \mathrm{~mL}$ iodized oil emulsion with 5 -fluorouracil $(500 \mathrm{mg})$, and/or epirubicin $(20 \mathrm{mg})$, and/or hydroxy camptothecin $(10 \mathrm{mg})$.

\section{Follow-up}

In the first 2 years after hepatectomy, the enrolled patients will be followed up every 2 months and then every 3 months until death or withdrawal from our research plan. All patients enrolled in this study were evaluated by basic medical history and physical examination at the discretion of the treating surgeons. Such as CEA, serum CA19-9, $\mathrm{AFP}$, liver function tests, and abdominal ultrasound were obtained at each of the follow-up visits. In both groups, follow-up contrast-enhanced computed tomography (CT) and/or magnetic resonance imaging (MRI) was performed once every 6 months or earlier if tumor recurrence was clinically suspected. Treating surgeon. In each followup, AFP, serum CA19-9, CEA and liver function tests and abdominal ultrasound were obtained. In the two groups, if tumor recurrence is suspected clinically, contrastenhanced computed tomography (CT) and/or magnetic resonance imaging (MRI) is performed every 6 months or earlier. In the case of recurrence, the treatment method was formulated according to the location, size and number of the recurring tumor and the liver function of patients.

\section{Statistical analysis}

OS and RFS were used as primary endpoints. OS was defined as the interval between hepatectomy and death, or the last date of follow-up program. RFS was defined as the time interval to tumor recurrence after previous hepatectomy or metastasis was diagnosed. Continuous data are expressed as median [interquartile range (IQR)] and compared with Mann-Whitney $U$ test; for comparing categorical variables, Fisher's exact test or Chi-square test was used. Firstly, clinicopathologic characteristics between patients with and without postoperative TACE were compared. Secondly, Kaplan-Meier survival curves were used to estimate the median, 1-, 3-, and 5-year OS and RFS, while the log-rank test was used to compare differences in 
Table 1 Baseline and clinicopathologic characteristics of patients with and without adjuvant TACE

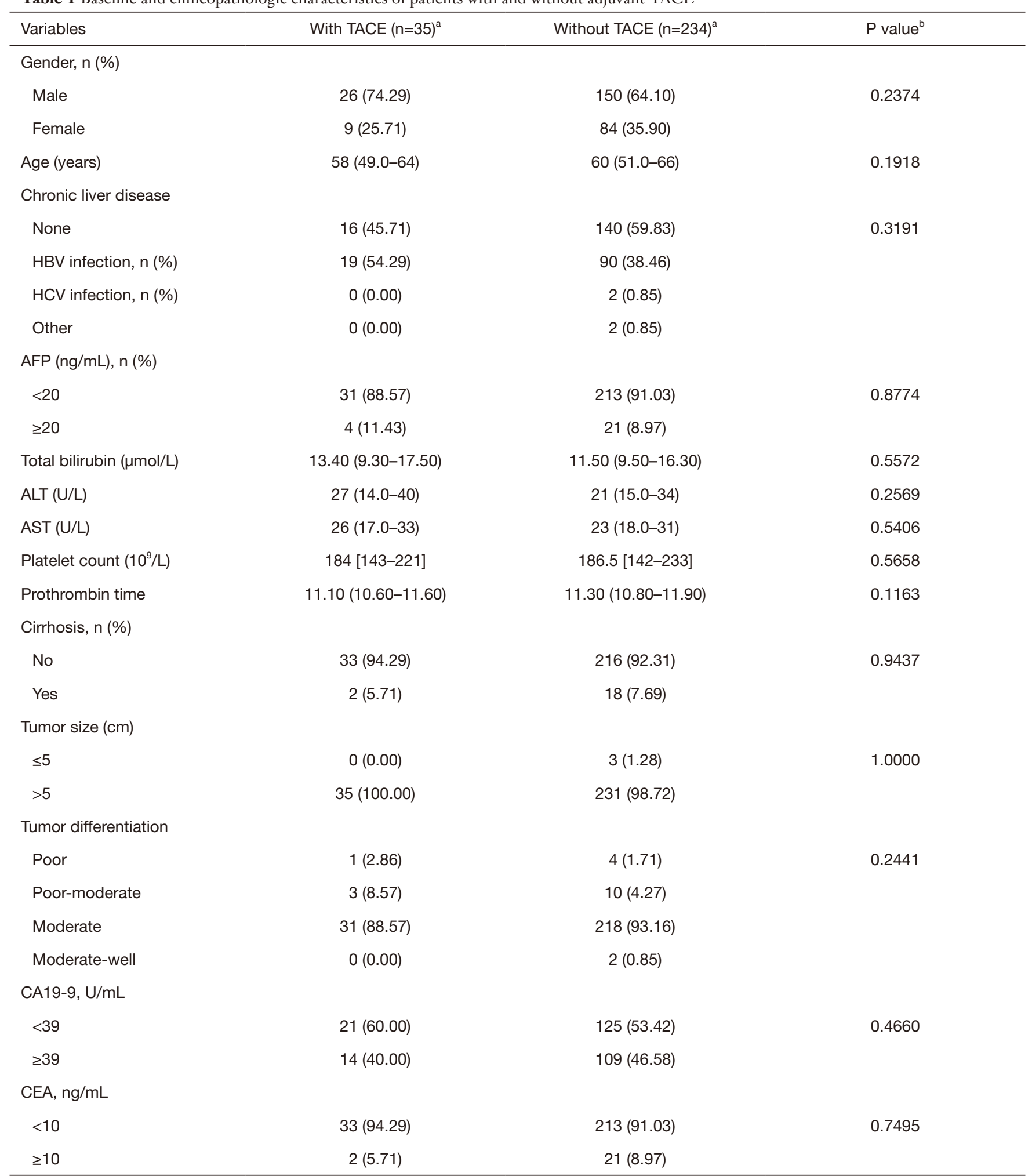

Table 1 (continued) 
Table 1 (continued)

\begin{tabular}{|c|c|c|c|}
\hline Variables & With TACE $(n=35)^{a}$ & Without TACE $(n=234)^{a}$ & $P$ value ${ }^{b}$ \\
\hline \multicolumn{4}{|l|}{ Tumor location } \\
\hline Left lobe & $22(62.86)$ & $106(45.30)$ & 0.1370 \\
\hline Right lobe & $13(37.14)$ & $123(52.56)$ & \\
\hline Caudate lobe & $0(0.00)$ & $4(1.71)$ & \\
\hline \multicolumn{4}{|l|}{ Nerve invasion } \\
\hline No & $34(97.14)$ & 217 (92.74) & 0.5414 \\
\hline Yes & $1(2.86)$ & $17(7.26)$ & \\
\hline
\end{tabular}

${ }^{a}$, data are expressed as median \pm interquartile range (IQR) or number (percentage); ${ }^{\mathrm{b}}$, based on Mann-Whitney $U$ test or chi-square test whenever appropriate. TACE, transarterial chemoembolization; HBV, hepatitis B virus; HCV, hepatitis C virus; AFP, alpha fetoprotein; ALT, alanine aminotransferase; AST, aspartate aminotransferase; CA19-9, carbohydrate antigen 19-9; CEA, carcinoembryonic antigen.

survival. The median follow-up time was estimated using the reverse Kaplan-Meier method. Factors were entered into the multivariable Cox proportional hazards regression model using backward stepwise selection with the Akaike information criterion (AIC). Hazard ratios (HRs) and 95\% CI were estimated. Statistical analyses were performed using SAS, version 9.4 (SAS Institute Inc), and R version 3.5.2 software packages (http://www.r-project.org/). Two sided $\mathrm{P}$ value $<0.05$ were considered statistically significant.

\section{Results}

\section{Baseline demographic and clinicopathologic characteristics}

In this retrospective analysis, we collected 269 patients diagnosed with TNM Stage I ICC received surgery from January 2012 to December 2016. Of the 269 patients included, the median age was 60.0 years (IQR, 51.066.0 years) and the majority of patients were male ( $\mathrm{n}=176$, 65.4\%). Overall, 109 (40.5\%) patients were concomitant with $\mathrm{HBV}$ infection, and 266 (98.9\%) patients had a tumor size of larger than $5 \mathrm{~cm}$. Most patients had moderately differentiated tumors $(249 ; 92.6 \%)$. Nerve invasion was noted in 18 (6.7\%) patients. Postoperative adjuvant TACE was carried out in 35 patients $(13.0 \%)$. The baseline demographic and clinicopathologic characteristics of patients with and without postoperative TACE were listed in Table 1. The differences in the baseline clinicopathologic features between the TACE group and the non-TACE group were statistically insignificant.

\section{OS and RFS}

The median follow-up time was 48.2 months (95\% CI: 45.17-51.07 months). Among all patients, the median OS was 66.8 months, and the 1-, 3-, and 5-year OS rates were $85.9 \%, 60.8 \%$, and $51.2 \%$, respectively (Table 2). At the last follow up, 119 patients had died. Median OS of patients with TACE was 59.9 months compared to 66.8 months in patients without TACE, which was not significant in univariate analysis $(\mathrm{P}=0.707)$ (Figure $1 A$ ). Tumor recurrence was detected in 138 patients $(51.3 \%)$ during follow up. The median RFS for all patients was 27.8 months, and the 1-, 3-, and 5-year recurrencefree rates were $67.7 \%, 45.0 \%$, and $32.9 \%$, respectively. Specifically, compared with patients with adjuvant TACE after hepatectomy, patients without adjuvant TACE had longer median RFS time (29.5 vs. 20.8 months) and higher $1-, 3$-, and 5-year RFS rate $(68.2 \%, 46.3 \%$, and $36.5 \%$, vs. $64.7 \%, 38.2 \%$, and NA) $(\mathrm{P}=0.0328)$ (Table 2 and Figure $1 B)$. Multivariable analysis was performed to assess the association between the use of TACE and without adjuvant TACE. Risk-adjustment revealed significant associations between OS and higher level of serum CA19-9, but no association with TACE procedure (Figure $1 C$ and Table 3). On multivariable analysis, adjuvant of TACE was an independent predictor of worse RFS (HR: 1.881, 95\% CI: 1.208-2.930) (Table 4 and Figure 1D). Risk-adjustment also revealed several significant prognostic factors associated with RFS, including chronic concomitant diseases, AFP, cirrhosis and CA19-9. 
Table 2 Median, 1-, 3-, and 5-year overall survival and recurrence-free survival in all patients and according to with TACE and without TACE groups

\begin{tabular}{|c|c|c|c|c|}
\hline Variable & All patients & With TACE & Without TACE & $P$ value \\
\hline \multicolumn{5}{|l|}{ Overall survival } \\
\hline Median (months) & 66.8 & 59.9 & 66.8 & \multirow[t]{3}{*}{0.7070} \\
\hline 3-year (\%) & 60.8 & 61.5 & 60.7 & \\
\hline 5-year (\%) & 51.2 & 49.2 & 51.1 & \\
\hline Median (months) & 27.8 & 20.8 & 29.5 & \multirow[t]{4}{*}{0.0328} \\
\hline 1-year (\%) & 67.7 & 64.7 & 68.2 & \\
\hline 3-year (\%) & 45.0 & 38.2 & 46.3 & \\
\hline 5-year (\%) & 32.9 & NA & 36.5 & \\
\hline
\end{tabular}

NA: not available because the observation with the largest recurrence-free survival time was censored and the estimation was restricted to the largest event time, which was less than 60 months. TACE, transarterial chemoembolization.
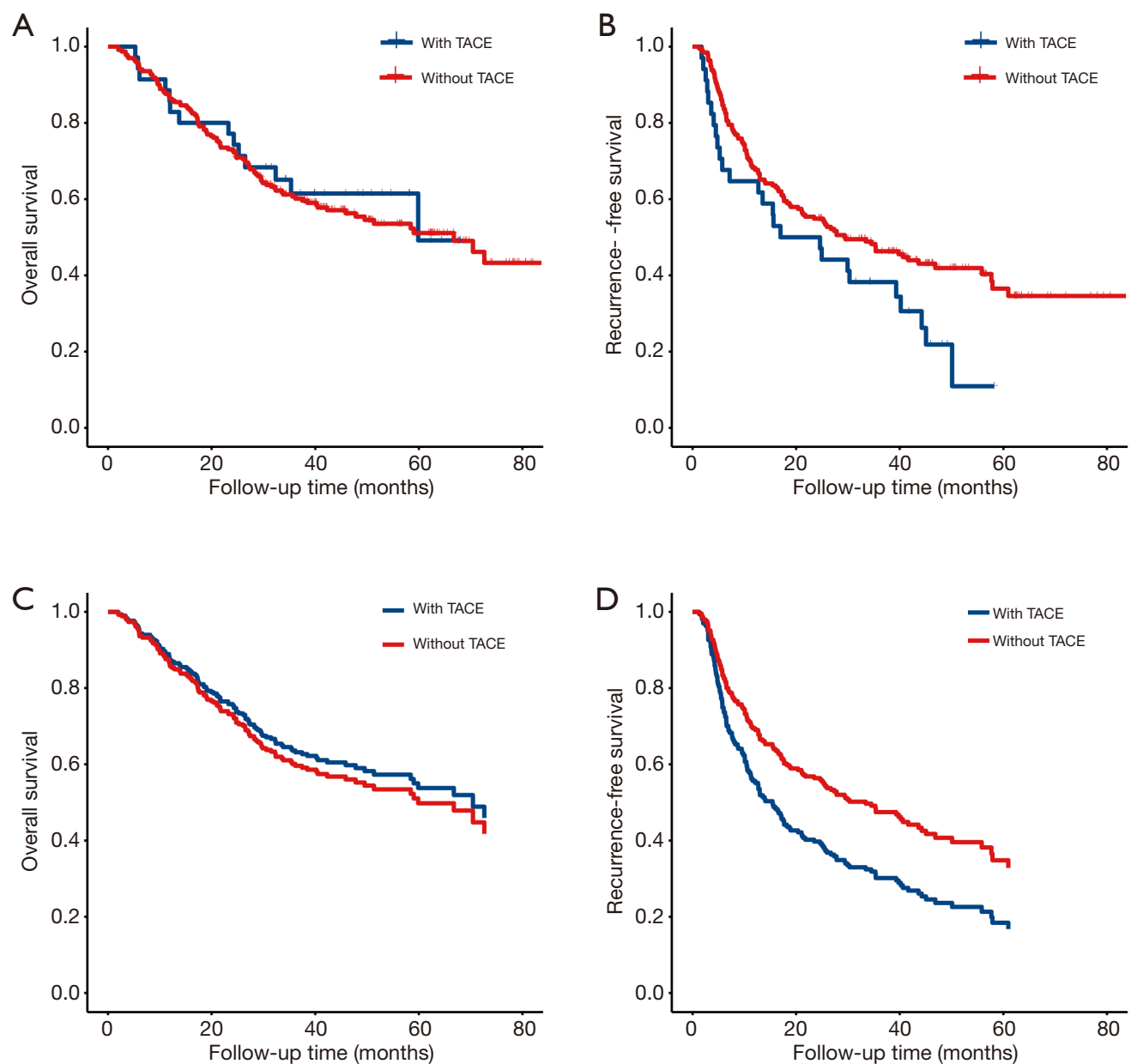

Figure 1 Comparison of overall survival and recurrence-free survival between the TACE and without TACE groups. (A,B) Crude overall survival and recurrence-free survival rate using Kaplan-Meier method. (C,D) Adjusted overall survival and recurrence-free survival rate using multivariable Cox proportional hazard model. TACE, transarterial chemoembolization. 
Table 3 Cox proportional hazards regression model showing the association of variables with overall survival (OS)

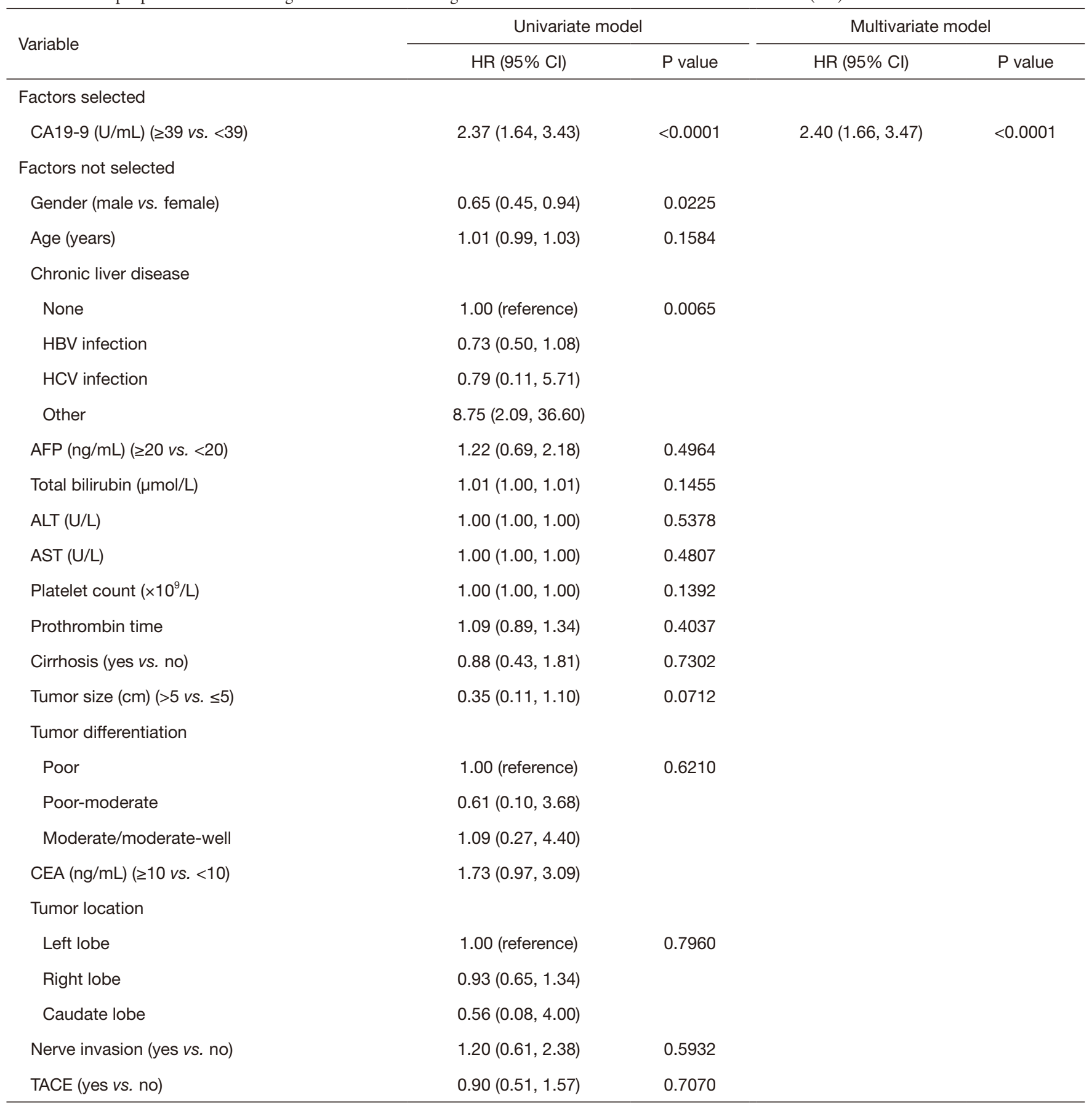

ICC, intrahepatic cholangiocarcinoma; HBV, hepatitis B virus; HCV, hepatitis C virus; AFP, alpha fetoprotein; ALT, alanine aminotransferase; AST, aspartate aminotransferase; CA19-9, carbohydrate antigen 19-9; CEA, carcinoembryonic antigen; TACE, transarterial chemoembolization; HR, hazard ratio; $\mathrm{Cl}$, confidence interval. 
Table 4 Cox proportional hazards regression model showing the association of variables with recurrence-free survival (RFS)

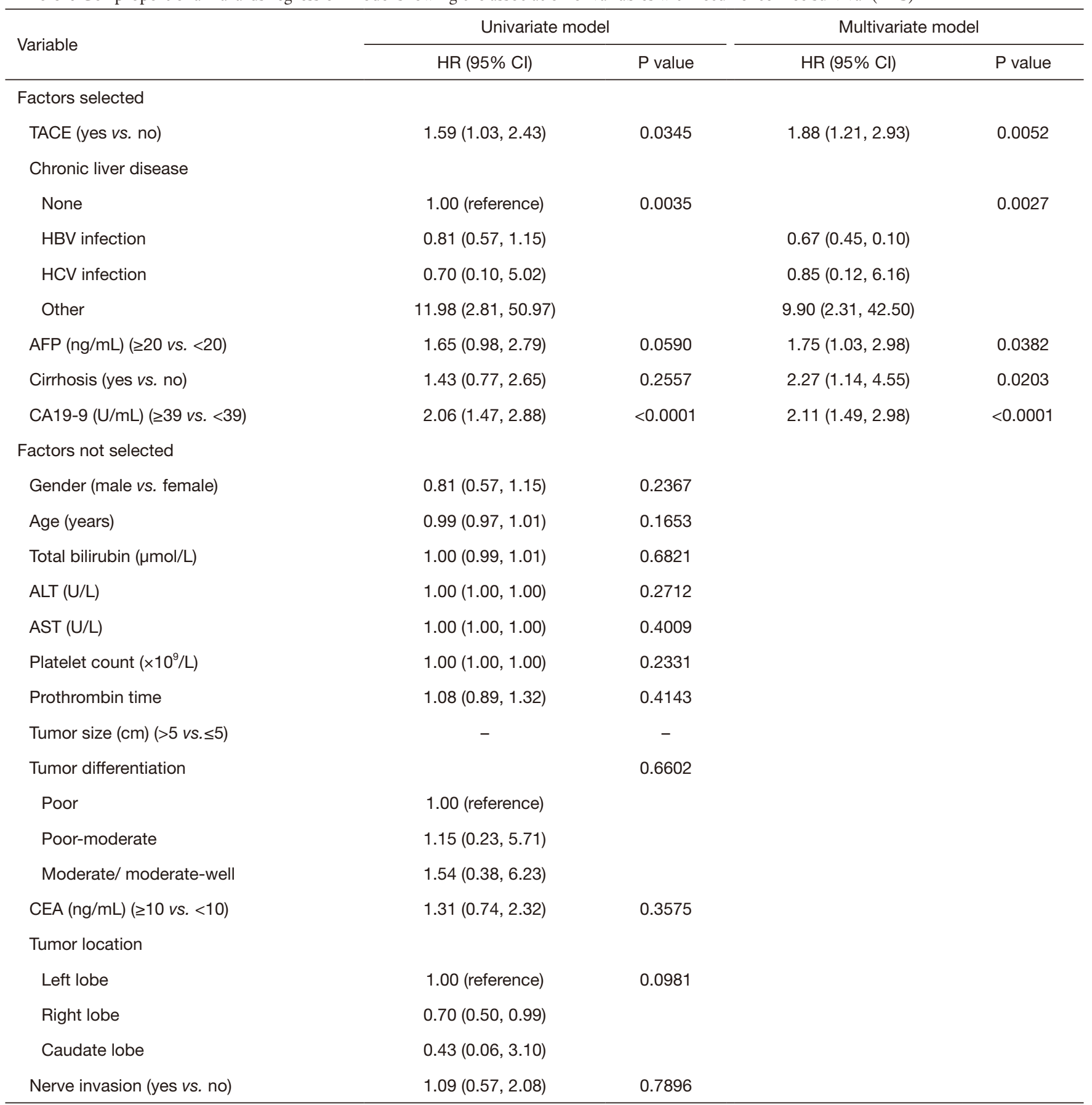

ICC, intrahepatic cholangiocarcinoma; HBV, hepatitis B virus; HCV, hepatitis C virus; AFP, alpha fetoprotein; ALT, alanine aminotransferase; AST, aspartate aminotransferase; CA19-9, carbohydrate antigen 19-9; CEA, carcinoembryonic antigen; TACE, transarterial chemoembolization; HR, hazard ratio; $\mathrm{Cl}$, confidence interval. 


\section{Discussion}

Among primary hepatic malignancies, ICC is the second most prevalent neoplasm after HCC (2), with the ageadjusted incidence increasing from 0.32 per 100,000 to 0.85 per 100,000 between 1975 and 2000 worldwide (17). Despite the progress of local-regional treatment, R0 resection provides the greatest long-term survival opportunity for ICC patients (2). Duo to delayed diagnosis, only $30-40 \%$ of patients with ICC (stage 1 and 2; TNM staging) are amenable to surgical resection (18). Unfortunately, even after $\mathrm{R} 0$ resection, the recurrence rate of the disease is still high, with some series reporting of $60 \%$ and $80 \%$ at a median follow-up of 21 months and 5 years, respectively $(19,20)$. ICC is related to shorter survival and lower resectability rates compared to other liver malignancies. Given the high recurrence rates observed in surgically treated ICC, postoperative adjuvant therapy seems to be very necessary. However, its exact role remains to be defined.

In clinical practice, multiple adjuvant and neoadjuvant therapies have been commonly conducted to reduce recurrence and improve the OS (21,22). Among these methods, TACE is regarded as an adjuvant way to prolong the OS after hepatectomy $(13,16,23)$. TACE is an effective adjuvant therapy for HCC patients after hepatectomy. A meta-analysis demonstrated that adjuvant TACE may suppress recurrence and improve survival rates following resection (24). TACE can effectively block the nutrient vessels of the invisible metastatic liver cancer and allowing sustainable chemotherapeutic killing of the microscopic HCC cells (25). Previous studies have shown that adjuvant TACE may benefit selected patients, but the sample size of these studies is small, and the roles of adjuvant TACE in patients with ICC after hepatectomy is still controversial.

Four articles reported the efficacy of adjuvant TACE after resection of ICC. One of the studies showed that adjuvant TACE did not delay disease recurrence, but prolonged OS in patients with early disease recurrence (13). Another study found that TACE can improve the survival rate of patients with ICC $>5 \mathrm{~cm}$ (15). In a study from Fudan University, 68 patients received postoperative TACE and 143 patients did not (23). Adjuvant TACE may be indicated only for patients at advanced stages for better survival. In another study, 122 patients with adjuvant TACE and 431 patients with $\mathrm{R} 0$ resection alone were analyzed (16). The 5-year recurrence rates were significantly lower after adjuvant TACE. Adjuvant TACE was associated with improved OS, the 5-year OS rates with and without TACE were $38 \%$ and $30 \%$, respectively. However, after 1:1 PSM, adjuvant TACE was not associated with higher OS or RFS. The results of the present study were consistent with these reports. In our study, of the 269 TNM stage I patients included, all of the patients underwent R0 resection. Adjuvant TACE was carried out in 35 patients. Among all patients, the median OS was 66.8 months, and the 1-, 3-, and 5-year OS rates were $85.9 \%, 60.8 \%$, and $51.2 \%$, respectively. Moreover, the median OS of patients with TACE was 59.9 months compared to 66.8 months in patients without TACE, which was not significant in univariate analysis $(\mathrm{P}=0.707)$.

In our study, the recurrence rate of TNM stage I ICC after R0 resection was still high, and the median RFS of all patients was 27.8 months. Patients without adjuvant TACE had longer median RFS time and higher 1-, 3-, and 5-year RFS rate compared with patients with adjuvant TACE after hepatectomy indicating that adjuvant TACE could not defer the recurrence. On multivariable analysis, the use of adjuvant of TACE was an independent predictor of worse RFS.

Our study has some limitations. This is a single-center, small sample-size retrospective cohort study, and the findings may be susceptible to unmeasured confounding. Further research from multicenter, randomized controlled trial with longer follow-up should be conducted in order to verify and extend the results in the present study.

In conclusion, postoperative adjuvant TACE does not appear to improve OS or reduce recurrence in patients with TNM stage I. On the contrary, adjuvant TACE may increase the risk of recurrence in ICC patients at TNM stage I.

\section{Acknowledgments}

Funding: This study was supported by the Natural Science Foundation of China (No. 81502093), the Natural Science Foundation of China (NSFC) grants (No. 81372672, 81672371), and Scientific Research Project of Shanghai Science and Technology Commission (No. 14411960200).

\section{Footnote}

Reporting Checklist: The authors have completed the STROBE reporting checklist. Available at http://dx.doi. org/10.21037/apm-20-1337

Data Sharing Statement: Available at http://dx.doi. 
org/10.21037/apm-20-1337

Conflicts of Interest: All authors have completed the ICMJE uniform disclosure form (available at http://dx.doi. org/10.21037/apm-20-1337). The authors have no conflicts of interest to declare.

Ethical Statement: The authors are accountable for all aspects of the work in ensuring that questions related to the accuracy or integrity of any part of the work are appropriately investigated and resolved. The study was conducted in accordance with the Declaration of Helsinki (as revised in 2013). The study was approved by the institutional ethics committee of the Eastern Hepatobiliary Surgery Hospital. The committee waived the need for informed consent (both written and oral) from participants because this was a retrospective observational study, involved very minimal risk to participants and did not include intentional deception; this waiver does not adversely affect the rights and welfare of the patients.

Open Access Statement: This is an Open Access article distributed in accordance with the Creative Commons Attribution-NonCommercial-NoDerivs 4.0 International License (CC BY-NC-ND 4.0), which permits the noncommercial replication and distribution of the article with the strict proviso that no changes or edits are made and the original work is properly cited (including links to both the formal publication through the relevant DOI and the license). See: https://creativecommons.org/licenses/by-nc-nd/4.0/.

\section{References}

1. Aljiffry M, Abdulelah A, Walsh M, et al. Evidence-based approach to cholangiocarcinoma: a systematic review of the current literature. J Am Coll Surg 2009;208:134-47.

2. Bridgewater J, Galle PR, Khan SA, et al. Guidelines for the diagnosis and management of intrahepatic cholangiocarcinoma. J Hepatol 2014;60:1268-89.

3. Wang Y, Li J, Xia Y, et al. Prognostic nomogram for intrahepatic cholangiocarcinoma after partial hepatectomy. J Clin Oncol 2013;31:1188-95.

4. Zhou H, Jiang X, Li Q, et al. A simple and effective prognostic staging system based on clinicopathologic features of intrahepatic cholangiocarcinoma. Am J Cancer Res 2015;5:1831-43.

5. Cillo U, Fondevila C, Donadon M, et al. Surgery for cholangiocarcinoma. Liver Int 2019;39 Suppl 1:143-55.
6. Spolverato G, Kim Y, Alexandrescu S, et al. Management and Outcomes of Patients with Recurrent Intrahepatic Cholangiocarcinoma Following Previous Curative-Intent Surgical Resection. Ann Surg Oncol 2016;23:235-43.

7. Chen X, Zhang B, Yin X, et al. Lipiodolized transarterial chemoembolization in hepatocellular carcinoma patients after curative resection. J Cancer Res Clin Oncol 2013;139:773-81.

8. Peng BG, He Q, Li JP, et al. Adjuvant transcatheter arterial chemoembolization improves efficacy of hepatectomy for patients with hepatocellular carcinoma and portal vein tumor thrombus. Am J Surg 2009;198:313-8.

9. Wang Z, Ren Z, Chen Y, et al. Adjuvant Transarterial Chemoembolization for HBV-Related Hepatocellular Carcinoma After Resection: A Randomized Controlled Study. Clin Cancer Res 2018;24:2074-81.

10. EASL-EORTC clinical practice guidelines: management of hepatocellular carcinoma. J Hepatol 2012;56:908-43.

11. Park SY, Kim JH, Yoon HJ, et al. Transarterial chemoembolization versus supportive therapy in the palliative treatment of unresectable intrahepatic cholangiocarcinoma. Clin Radiol 2011;66:322-8.

12. Ray CE, Jr., Edwards A, Smith MT, et al. Metaanalysis of survival, complications, and imaging response following chemotherapy-based transarterial therapy in patients with unresectable intrahepatic cholangiocarcinoma. J Vasc Interv Radiol 2013;24:1218-26.

13. Shen WF, Zhong W, Liu Q, et al. Adjuvant transcatheter arterial chemoembolization for intrahepatic cholangiocarcinoma after curative surgery: retrospective control study. World J Surg 2011;35:2083-91.

14. Jeong S, Zheng B, Wang J, et al. Transarterial Chemoembolization: A Favorable Postoperative Management to Improve Prognosis of Hepatitis B Virusassociated Intrahepatic Cholangiocarcinoma after Surgical Resection. Int J Biol Sci 2017;13:1234-41.

15. Wu ZF, Zhang HB, Yang N, et al. Postoperative adjuvant transcatheter arterial chemoembolisation improves survival of intrahepatic cholangiocarcinoma patients with poor prognostic factors: results of a large monocentric series. Eur J Surg Oncol 2012;38:602-10.

16. Li J, Wang Q, Lei Z, et al. Adjuvant Transarterial Chemoembolization Following Liver Resection for Intrahepatic Cholangiocarcinoma Based on Survival Risk Stratification. Oncologist 2015;20:640-7.

17. Shaib YH, Davila JA, McGlynn K, et al. Rising incidence of intrahepatic cholangiocarcinoma in the United States: a true increase? J Hepatol 2004;40:472-7. 
18. Weber SM, Ribero D, O'Reilly EM, et al. Intrahepatic cholangiocarcinoma: expert consensus statement. HPB (Oxford) 2015;17:669-80.

19. Hyder O, Hatzaras I, Sotiropoulos GC, et al. Recurrence after operative management of intrahepatic cholangiocarcinoma. Surgery 2013;153:811-8.

20. Tabrizian P, Jibara G, Hechtman JF, et al. Outcomes following resection of intrahepatic cholangiocarcinoma. HPB (Oxford) 2015;17:344-51.

21. Buettner S, van Vugt JL, JN IJ, et al. Intrahepatic cholangiocarcinoma: current perspectives. Onco Targets Ther 2017;10:1131-42.

22. Le Roy B, Gelli M, Pittau G, et al. Neoadjuvant

Cite this article as: Liu G, Guo W, Wang H, Liu W, Lei L, Xie Q, Li X, Zou S, Wang P, Zhou H, Hu H. Influence of postoperative adjuvant transarterial chemoembolization on the prognosis of early-stage intrahepatic cholangiocarcinoma: a single center study. Ann Palliat Med 2021;10(4):3673-3683. doi: 10.21037/apm-20-1337 chemotherapy for initially unresectable intrahepatic cholangiocarcinoma. Br J Surg 2018;105:839-47.

23. Li T, Qin LX, Zhou J, et al. Staging, prognostic factors and adjuvant therapy of intrahepatic cholangiocarcinoma after curative resection. Liver Int 2014;34:953-60.

24. Zhong JH, Li LQ. Postoperative adjuvant transarterial chemoembolization for participants with hepatocellular carcinoma: A meta-analysis. Hepatol Res 2010;40:943-53.

25. Roayaie S, Frischer JS, Emre SH, et al. Long-term results with multimodal adjuvant therapy and liver transplantation for the treatment of hepatocellular carcinomas larger than 5 centimeters. Ann Surg 2002;235:533-9. 